PROCEEDINGS OF THE

AMERICAN MATHEMATICAL SOCIETY

Volume 133, Number 2, Pages 369-377

S 0002-9939(04)07510-0

Article electronically published on August 26, 2004

\title{
ISOMETRIES FOR KY FAN NORMS BETWEEN MATRIX SPACES
}

\author{
CHI-KWONG LI, YIU-TUNG POON, AND NUNG-SING SZE
}

(Communicated by Joseph A. Ball)

\begin{abstract}
We characterize linear maps between different rectangular matrix spaces preserving Ky Fan norms.
\end{abstract}

\section{INTRODUCTION AND STATEMENTS OF RESULTS}

Let $M_{m, n}\left(M_{n}\right)$ be the linear space of $m \times n(n \times n)$ complex matrices. The singular values of $A \in M_{m, n}$ are the nonnegative square roots of the eigenvalues of $A^{*} A$, and they are denoted by $s_{1}(A) \geq \cdots \geq s_{n}(A)$. For $1 \leq k \leq \min \{m, n\}$, the Ky Fan $k$-norm on $M_{m, n}$ is defined and denoted by

$$
\|A\|_{k}=s_{1}(A)+\cdots+s_{k}(A) .
$$

The Ky Fan 1-norm reduces to the operator norm; when $m=n$ the Ky Fan $n$-norm is also known as the trace norm.

Evidently, Ky Fan $k$-norms are unitarily invariant norms, i.e.,

$$
\|U A V\|_{k}=\|A\|_{k}
$$

for any $A \in M_{m, n}$, and unitary $U \in M_{m}$ and $V \in M_{n}$. Actually, they form an important class of unitarily invariant norms; see [1, Chapters 2 and 3]. For instance, given $A, B \in M_{m, n}$,

$$
\|A\|_{k} \leq\|B\|_{k} \quad \text { for all } k=1, \ldots, \min \{m, n\}
$$

if and only if

$$
\|A\| \leq\|B\| \quad \text { for all unitarily invariant norms }\|\cdot\| .
$$

There has been considerable interest in studying isometries for Ky Fan norms on matrix spaces. For example, by a result of Kadison [5], one easily deduces that isometries for the operator norm on $M_{n}$ have to have the form

$$
A \mapsto U A V \quad \text { or } \quad A \mapsto U A^{t} V
$$

for some unitary matrices $U, V \in M_{n}$. In [4], the authors showed that the same conclusion holds for Ky Fan $k$-norm isometries for any $k=1, \ldots, \min \{m, n\}$, where the second form in (1) can occur only when $m=n$. In [8, the authors considered the

Received by the editors July 8, 2003 and, in revised form, August 26, 2003.

2000 Mathematics Subject Classification. Primary 15A04, 15A60.

Key words and phrases. Isometry, matrices, linear maps.

The first author's research was partially supported by NSF.

The third author thanks Dr. Jor-Ting Chan for his guidance and encouragement. 
problem on block triangular matrix algebras in $M_{n}$, and showed that the isometries essentially have the same structure. In [3], the authors studied isometries $\phi$ : $\left(M_{n},\|\cdot\|_{1}\right), \rightarrow\left(M_{p},\|\cdot\|_{1}\right)$ for $n \neq p$, and obtained a complete characterization when $p \leq 2 n-2$; moreover, examples were given to show that $\phi$ may have complicated structure for $p>2 n-2$. In view of these, one may think that isometries $\phi$ : $\left(M_{n},\|\cdot\|_{k}\right), \rightarrow\left(M_{p},\|\cdot\|_{k}\right)$ also have complicated structure for $k>1$. It turns out that it is not the case as shown in the corollary of our main theorem, which characterizes isometries $\phi:\left(M_{m, n},\|\cdot\|_{k^{\prime}}\right), \rightarrow\left(M_{p, q},\|\cdot\|_{k}\right)$ provided $k^{\prime}>1$. We need some notation and definitions to describe our main result.

For two matrices $A$ and $B$ with $A=\left(a_{i j}\right)$ denote by $A \otimes B$ the block matrix $\left(a_{i j} B\right)$. An $r \times s$ matrix $X$ is called a partial isometry if $X^{*} X=I_{s}$, i.e., $X$ has orthonormal columns.

Theorem 1.1. Let $1<k^{\prime} \leq \min \{m, n\}$ and $1 \leq k \leq \min \{p, q\}$. Suppose $\phi$ : $M_{m, n} \rightarrow M_{p, q}$ satisfies

$$
\|\phi(A)\|_{k}=\|A\|_{k^{\prime}} \quad \text { for all } \quad A \in M_{m, n} .
$$

Then there exist nonnegative integers $c_{1}$ and $c_{2}$ with $c_{1}+c_{2}>0$, and partial isometries $U$ and $V$ of sizes $p \times\left(c_{1} m+c_{2} n\right)$ and $q \times\left(c_{1} n+c_{2} m\right)$, respectively, such that one of the following holds:

(a) $k^{\prime}<\min \{m, n\}, k=k^{\prime}\left(c_{1}+c_{2}\right)$, and $\phi$ has the form

$$
\left.A \mapsto \frac{1}{c_{1}+c_{2}} U\left[\left(I_{c_{1}} \otimes A\right) \oplus\left(I_{c_{2}} \otimes A\right)^{t}\right)\right] V^{*} .
$$

(b) $k^{\prime}=\min \{m, n\}, k^{\prime}\left(c_{1}+c_{2}\right) \leq k$, and there are diagonal matrices $D_{1} \in M_{c_{1}}$ and $D_{2} \in M_{c_{2}}$ with positive diagonal entries satisfying $\operatorname{tr} D_{1}+\operatorname{tr} D_{2}=1$, such that $\phi$ has the form

$$
A \mapsto U\left[\left(D_{1} \otimes A\right) \oplus\left(D_{2} \otimes A^{t}\right)\right] V^{*} .
$$

If $k^{\prime}=k$, then either $\left(c_{1}, c_{2}\right)=(1,0)$ or $\left(c_{1}, c_{2}\right)=(0,1)$. By adding columns to $U$ and $V$ to form unitary matrices, we have the following corollary.

Corollary 1.2. Let $1<k \leq \min \{m, n\}$. Suppose $\phi: M_{m, n} \rightarrow M_{p, q}$ satisfies

$$
\|\phi(A)\|_{k}=\|A\|_{k} \quad \text { for all } \quad A \in M_{m, n} .
$$

Then there are unitary matrices $U \in M_{p}$ and $V \in M_{q}$ such that $\phi$ has the form

$$
A \mapsto U\left[A \oplus 0_{p-m, q-n}\right] V \quad \text { or } \quad A \mapsto U\left[A^{t} \oplus 0_{p-n, q-m}\right] V .
$$

\section{AuXiliary Results AND PROOFS}

Replacing $\phi$ by the mapping(s) $A \mapsto \phi\left(A^{t}\right)$ and/or $A \mapsto[\phi(A)]^{t}$, we may assume that $m \leq n$ and $p \leq q$. Two nonzero matrices $A, B \in M_{m, n}$ are said to be orthogonal if $A B^{*}=0$ and $A^{*} B=0$. Equivalently, there are unitary matrices $U$ and $V$ such that $U A V=\sum_{j=1}^{r} a_{j} E_{j j}$ and $U B V=\sum_{j=r+1}^{r+s} b_{j} E_{j j}$ with $a_{1} \geq \cdots \geq a_{r}>0$ and $b_{1} \geq \cdots \geq b_{s}>0$ for some $r, s$ with $r+s \leq \min \{m, n\}$. The nonzero matrices $A_{1}, \cdots A_{d} \in M_{m, n}$ are said to be pairwise orthogonal $m \times n$ matrices if $A_{i} A_{j}^{*}=0$ and $A_{i}^{*} A_{j}=0$ for any distinct pair $(i, j)$. In such a case, there are unitary $U \in M_{m}$ and $V \in M_{n}, 0=r_{0}<r_{1}<\cdots<r_{d} \leq \min \{m, n\}$ and positive numbers $a_{1}, \ldots, a_{r_{d}}$ such that $U A_{i} V=\sum_{r_{i-1}<j \leq r_{i}} a_{j} E_{j j}$. 
We begin with the following lemma from [8, Lemma 5].

Lemma 2.1. Let $A, B \in M_{m, n}$ be nonzero. Then $\|a A+b B\|_{k}=|a|\|A\|_{k}+|b|\|B\|_{k}$ for every $a, b \in \mathbb{C}$ if and only if $A$ and $B$ are orthogonal and $\operatorname{rank} A+\operatorname{rank} B \leq k$.

By Lemma 2.1 and a simple inductive argument, we have the following.

Lemma 2.2. Let $\phi: M_{m, n} \rightarrow M_{p, q}$ be a map satisfying (2). Suppose the rank one matrices $A_{1}, \ldots, A_{d} \in M_{m, n}, d \leq \min \{m, n\}$, are pairwise orthogonal. Then $\phi\left(A_{1}\right), \ldots, \phi\left(A_{d}\right) \in M_{p, q}$ are nonzero and pairwise orthogonal. Furthermore, for any $1 \leq s_{1}<\cdots<s_{k^{\prime}} \leq d, \sum_{j=1}^{k^{\prime}} \operatorname{rank} \phi\left(A_{s_{j}}\right) \leq k$.

Proof of Theorem 1.1. For the sufficiency part of Theorem 1.1, one readily sees that singular values of $\phi(A)$ have $c=\left(c_{1}+c_{2}\right)$ copies of $s_{1}(A) / c, \ldots, s_{m}(A) / c$ if $\phi$ has the form $(a)$. On the other hand, if $k^{\prime}=m$ and $\phi$ has the form $(b)$, then $k \geq c k^{\prime}$ and so the Ky Fan $k$-norm of $\phi(A)$ is just the sum of its singular values. Let $D_{1} \oplus D_{2}=\operatorname{diag}\left(d_{1}, \ldots, d_{c}\right)$. Then,

$$
\|\phi(A)\|_{k}=d_{1}\|A\|_{k^{\prime}}+\cdots+d_{c}\|A\|_{k^{\prime}}=\operatorname{tr}\left(D_{1} \oplus D_{2}\right)\|A\|_{k^{\prime}}=\|A\|_{k^{\prime}} .
$$

To prove the necessity part, let $\left(p^{\prime}, q^{\prime}\right)=\left(p-c_{1} m-c_{2} n, q-c_{1} n-c_{2} m\right)$. It suffices to prove that there are unitary matrices $U \in M_{p}$ and $V \in M_{q}$ such that $\phi$ has the form

$$
\begin{aligned}
& A \mapsto \frac{1}{c_{1}+c_{2}} U\left[\left(I_{c_{1}} \otimes A\right) \oplus\left(I_{c_{2}} \otimes A^{t}\right) \oplus 0_{p^{\prime}, q^{\prime}}\right] V^{*} \quad \text { if } \quad k^{\prime}<m, \\
& A \mapsto U\left[\left(D_{1} \otimes A\right) \oplus\left(D_{2} \otimes A^{t}\right) \oplus 0_{p^{\prime}, q^{\prime}}\right] V^{*} \quad \text { if } \quad k^{\prime}=m .
\end{aligned}
$$

We divide the proof into three cases:

$$
\text { (I) } k^{\prime}<m=n, \quad \text { (II) } k^{\prime}=m=n, \quad \text { and } \quad \text { (III) } m<n \text {. }
$$

First consider case (I) $: k^{\prime}<m=n$. For any $A \in M_{m, n}$ with singular values $1,0, \ldots, 0$, there are unitary $X$ and $Y$ such that $A=X E_{11} Y$. Let $A_{j}=X E_{j j} Y$ for $j=1, \ldots, m$. Then $A_{1}, \ldots, A_{m}$ are pairwise orthogonal. By Lemma 2.2 $\phi\left(A_{1}\right), \ldots, \phi\left(A_{m}\right)$ are pairwise orthogonal. Thus, there exist unitary $U$ and $V$, $0=r_{0}<r_{1}<\cdots<r_{d} \leq m$ and positive numbers $a_{1}, \ldots, a_{r_{d}}$ such that

$$
B_{i}=U \phi\left(A_{i}\right) V=\sum_{r_{i-1}<j \leq r_{i}} a_{j} E_{j j} \quad \text { for any } \quad i=1, \ldots, m .
$$

By Lemma 2.2 again, the sum of any $k^{\prime}$ matrices chosen from $B_{1}, \ldots, B_{m}$ has rank at most $k$. Let $1 \leq t_{1}<\cdots<t_{k^{\prime}} \leq m$. Then

$$
s_{\ell}\left(\sum_{j=1}^{k^{\prime}} B_{t_{j}}\right)=0, \text { for all } \ell>k .
$$

Moreover, if $t \in\{1, \ldots, m\} \backslash\left\{t_{1}, \ldots, t_{k^{\prime}}\right\}$, we claim that

$$
s_{1}\left(B_{t}\right) \leq s_{k}\left(\sum_{j=1}^{k^{\prime}} B_{t_{j}}\right) \text {. }
$$

If (44) does not hold, then $s_{1}\left(B_{t}\right)>s_{k}\left(\sum_{j=1}^{k^{\prime}} B_{t_{j}}\right)$, which gives the following contradiction:

$$
k^{\prime}=\left\|A_{t}+\sum_{j=1}^{k^{\prime}} A_{t_{j}}\right\|_{k^{\prime}}=\left\|B_{t}+\sum_{j=1}^{k^{\prime}} B_{t_{j}}\right\|_{k}>\left\|\sum_{j=1}^{k^{\prime}} B_{t_{j}}\right\|_{k}=\left\|\sum_{j=1}^{k^{\prime}} A_{t_{j}}\right\|_{k^{\prime}}=k^{\prime} .
$$


Let $c=k / k^{\prime}$. It follows from (21), (3) and (44) that for each $1 \leq j \leq m$, we have $s_{i}\left(B_{j}\right)=1 / c$ for $1 \leq i \leq c$ and $s_{i}\left(B_{j}\right)=0$ for $c<i \leq p$. Thus, we see that

(i) every rank one matrix is mapped to a rank $c$ matrix, and

(ii) every unitary matrix is mapped to a matrix with the singular values $\underbrace{1 / c, \ldots, 1 / c}_{c m}, 0, \ldots, 0$.

Since (i) holds, by Theorem 2.5 in [7], $\phi$ has the form

$$
A \mapsto R\left[\left(I_{c_{1}} \otimes A\right) \oplus\left(I_{c_{2}} \otimes A^{t}\right) \oplus 0_{p^{\prime}, q^{\prime}}\right] S^{*}
$$

for some invertible $R \in M_{p}$ and $S \in M_{q}$. Let $R_{1}$ (respectively, $S_{1}$ ) be obtained from $R$ (respectively, $S$ ) by removing its last $p^{\prime}$ (respectively, $q^{\prime}$ ) columns. Then

$$
R\left[\left(I_{c_{1}} \otimes A\right) \oplus\left(I_{c_{2}} \otimes A^{t}\right) \oplus 0_{p^{\prime}, q^{\prime}}\right] S^{*}=R_{1}\left[\left(I_{c_{1}} \otimes A\right) \oplus\left(I_{c_{2}} \otimes A^{t}\right)\right] S_{1}^{*} .
$$

By polar decomposition, there are unitary matrices $U \in M_{p}, V \in M_{q}$ and positive definite matrices $P \in M_{c_{1} m+c_{2} n}$ and $Q \in M_{c_{1} n+c_{2} m}$ such that

$$
R_{1}=U\left(\begin{array}{c}
P \\
0_{p^{\prime}, c_{1} m+c_{2} n}
\end{array}\right) \quad \text { and } \quad S_{2}=V\left(\begin{array}{c}
Q \\
0_{q^{\prime}, c_{1} n+c_{2} m}
\end{array}\right) .
$$

Thus,

$$
\phi(A)=U\left\{P\left[\left(I_{c_{1}} \otimes A\right) \oplus\left(I_{c_{2}} \otimes A^{t}\right)\right] Q^{*} \oplus 0_{p^{\prime}, q^{\prime}}\right\} V^{*} .
$$

Define $\psi: M_{m} \rightarrow M_{c m}$ such that $\psi(X)=c P\left[\left(I_{c_{1}} \otimes A\right) \oplus\left(I_{c_{2}} \otimes A^{t}\right)\right] Q^{*}$. By (ii), we see that $\psi$ maps unitary matrices to unitary matrices. By the result in [2], we see that $\psi(A)=W_{1}\left[\left(I_{c_{1}} \otimes A\right) \oplus\left(I_{c_{2}} \otimes A^{t}\right)\right] W_{2}$ for some unitary $W_{1}, W_{2} \in M_{c m}$. Thus, condition (a) holds.

Next, we turn to case (II) : $k^{\prime}=m=n$. From the first part of the proof in case (I), we can see that for any unitary $X, Y \in M_{m}$ and $\lambda_{1}, \ldots, \lambda_{m} \in \mathbb{C}$, $\sum_{i=1}^{m} \lambda_{i} \phi\left(X E_{i i} Y\right)$ has rank at most $k$. Hence, $\phi(A)$ has rank at most $k$ for all $A \in M_{m}$. We may assume that $p=q$ by appending $q-p$ zero rows to $\phi(A)$ for each $A \in M_{m}$. So, we assume that $\phi: M_{m} \rightarrow M_{p}$ and suppose $\phi\left(I_{m}\right)=D$ is a nonnegative diagonal matrix with diagonal entries arranged in descending order. For any Hermitian $X \in M_{m}$ with trace zero and spectrum in $[-1,1]$ and $t \in[-1,1]$,

$$
\left\|\phi\left(I_{m}+t X\right)\right\|_{k}=\left\|I_{m}+t X\right\|_{k^{\prime}}=k^{\prime}=\left\|I_{m}\right\|_{k^{\prime}}=\left\|\phi\left(I_{m}\right)\right\|_{k}=\operatorname{tr} D .
$$

Let $Y=\phi(X)$. Then $\operatorname{tr} Y=0$ because

$$
|\operatorname{tr} D+t \operatorname{tr} Y| \leq\left\|\phi\left(I_{m}+t X\right)\right\|_{p}=\left\|\phi\left(I_{m}+t X\right)\right\|_{k}=\operatorname{tr} D
$$

for $t= \pm 1$. Moreover,

$$
k^{\prime}=\operatorname{tr}(D \pm Y) \leq\left\|\phi\left(I_{m}+t X\right)\right\|_{p}=\left\|\phi\left(I_{m}+t X\right)\right\|_{k}=k^{\prime} .
$$

By [6, Corollary 3.2], we conclude that $D \pm Y$ is positive semidefinite. As a result, if $\phi\left(I_{m}\right)=D=\operatorname{diag}\left(d_{1}, \ldots, d_{r}, 0, \ldots, 0\right)$ with $d_{1} \geq \cdots \geq d_{r}>0$, then $\phi(X)$ has the form $Y \oplus 0_{p-r}$. We may now consider $\psi: M_{m} \rightarrow M_{r}$ such that $\phi(A)=\psi(A) \oplus 0_{p-r}$. It follows from the above argument that $\psi$ maps Hermitian matrices to Hermitian matrices and $\|\psi(A)\|_{r}=\|\phi(A)\|_{k}=\|A\|_{k^{\prime}}$. We claim that

(i) $\psi$ maps positive semidefinite matrices to positive semidefinite matrices, and

(ii) $\psi$ maps invertible Hermitian matrices to invertible Hermitian matrices. 
To see (i), suppose that $A \in M_{m}$ is positive semidefinite. Let $D_{1}=\psi\left(I_{m}\right)=$ $\operatorname{diag}\left(d_{1}, \ldots, d_{r}\right)$. Choose $t>0$ such that $D_{1}+t \psi(A)$ is positive semidefinite. Then we have

$$
\begin{aligned}
\operatorname{tr}\left(D_{1}+t \psi(A)\right) & =\left\|D_{1}+t \psi(A)\right\|_{r}=\left\|I_{m}+t A\right\|_{k^{\prime}}=\operatorname{tr}\left(I_{m}\right)+t \operatorname{tr}(A) \\
& =\left\|I_{m}\right\|_{k^{\prime}}+t\|A\|_{k^{\prime}}=\left\|\psi\left(I_{m}\right)\right\|_{r}+t\|\psi(A)\|_{r}=\operatorname{tr} D_{1}+t\|\psi(A)\|_{r} .
\end{aligned}
$$

Thus, $\operatorname{tr} \psi(A)=\|\psi(A)\|_{r}$, and it follows from [6, Corollary 3.2] again that $\psi(A)$ is positive semidefinite.

To prove (ii), let

$$
A=U^{*}\left(\sum_{j=1}^{m} \lambda_{j} E_{j j}\right) U
$$

for some unitary $U$ and $\lambda_{j} \in \mathbb{R} \backslash\{0\}$ for $j=1, \ldots, m$. Since $\phi\left(U^{*} E_{11} U\right), \ldots$, $\phi\left(U^{*} E_{m m} U\right)$ are pairwise orthogonal and $\phi\left(I_{m}\right)=D, \phi\left(U^{*} E_{j j} U\right)=V^{*} F_{j} V \oplus 0_{p-r}$ for $j=1, \ldots, m$, such that $F_{i}=\sum_{r_{i-1}<s \leq r_{i}} a_{s} E_{s s}$ for $0=r_{0}<\cdots<r_{m}=r$ and positive numbers $a_{1}, \ldots, a_{r_{m}}$. Therefore,

$$
\psi(A)=V^{*}\left(\sum_{j=1}^{m} \lambda_{j}\left(\sum_{r_{i-1}<s \leq r_{i}} a_{s} E_{s s}\right)\right) V
$$

is also invertible. Thus, condition (ii) holds.

Now, $\psi\left(I_{m}\right)$ is positive definite and $\psi$ maps invertible Hermitian matrices to invertible Hermitian matrices. By (the proof of) [7, Proposition 3.4], we see that

$$
\psi(X)=T^{*}\left[\left(I_{c_{1}} \otimes X\right) \oplus\left(I_{c_{2}} \otimes X^{t}\right)\right] T
$$

for some invertible $T \in M_{r}$. In particular, we see that

(iii) $\psi$ maps rank $s$ matrices to rank cs matrices for $s=1, \ldots, m$.

Next, we show that $\psi$ has the form $X \mapsto U^{*}\left[\left(D_{1} \otimes X\right) \oplus\left(D_{2} \otimes X^{t}\right)\right] U$ for some unitary matrix $U$ and diagonal matrices $D_{1}$ and $D_{2}$ with positive diagonal entries such that $\operatorname{tr} D_{1}+\operatorname{tr} D_{2}=1$. Equivalently, we show that $\psi$ has the form

$$
A=\left(a_{u v}\right) \longmapsto V^{*} B V \text {, where } B=\left(B_{u v}\right)_{1 \leq u, v \leq m} \text { with } B_{u v}=a_{u v} D_{1} \oplus a_{v u} D_{2}
$$

for some unitary $V$. First, by a suitable permutation, we can rewrite $\psi$ in (5) as

$$
A=\left(a_{u v}\right) \longmapsto S^{*} B S \text { with } B=\left(B_{u v}\right)_{1 \leq u, v \leq m} \text { with } B_{u v}=a_{u v} I_{c_{1}} \oplus a_{v u} I_{c_{2}}
$$

for some nonsingular $S \in M_{r}$. By Lemma 2.2 , we see that $\phi\left(E_{11}\right), \ldots, \phi\left(E_{m m}\right)$ are pairwise orthogonal. Then for any distinct pair $i$ and $j$,

$$
\left[S^{*}\left(E_{i i} \otimes I_{c}\right) S\right]^{*}\left[S^{*}\left(E_{j j} \otimes I_{c}\right) S\right]=\psi\left(E_{i i}\right)^{*} \psi\left(E_{j j}\right)=0 .
$$

Thus, $\left(E_{i i} \otimes I_{c}\right) S S^{*}\left(E_{j j} \otimes I_{c}\right)=0$ whenever $i \neq j$. It follows that $S S^{*}=S_{1} \oplus \cdots \oplus S_{n}$ where $S_{i} \in M_{c}$.

Let $i>1, X=E_{11}+E_{1 i}$ and $Y=E_{i 1}-E_{i i}$. From (6), $\psi(X)=S^{*}\left(B_{r s}\right) S$ and $\psi(Y)=S^{*}\left(C_{r s}\right) S$ so that

$$
\begin{aligned}
\tilde{B} & =\left(\begin{array}{ll}
B_{11} & B_{1 i} \\
B_{i 1} & B_{i i}
\end{array}\right)=\left(\begin{array}{cc}
I_{c} & I_{c_{1}} \oplus 0_{c_{2}} \\
0_{c_{1}} \oplus I_{c_{2}} & 0_{c}
\end{array}\right), \\
\tilde{C} & =\left(\begin{array}{ll}
C_{11} & C_{1 i} \\
C_{i 1} & C_{i i}
\end{array}\right)=\left(\begin{array}{cc}
0_{c} & 0_{c_{1}} \oplus I_{c_{2}} \\
I_{c_{1}} \oplus 0_{c_{2}} & -I_{c}
\end{array}\right)
\end{aligned}
$$


and all other $B_{u v}$ and $C_{u v}$ are $0_{c}$. Let $J_{1}=I_{c_{1}} \oplus 0_{c_{2}}$ and $J_{2}=0_{c_{1}} \oplus I_{c_{2}}$. Since $X$ and $Y$ are orthogonal, so are $\psi(X)$ and $\psi(Y)$. Hence $B^{*}\left(S S^{*}\right) C=0$ and $B\left(S S^{*}\right) C^{*}=0$. Thus,

$$
\begin{aligned}
\left(\begin{array}{cc}
J_{2} S_{i} J_{1} & S_{1} J_{2}-J_{2} S_{i} \\
0 & J_{1} S_{1} J_{2}
\end{array}\right) & =\tilde{B}^{*}\left(S_{1} \oplus S_{i}\right) \tilde{C}=0 \\
& =\tilde{B}\left(S_{1} \oplus S_{i}\right) \tilde{C}^{*}=\left(\begin{array}{cc}
J_{1} S_{i} J_{2} & S_{1} J_{1}-J_{1} S_{i} \\
0 & J_{2} S_{1} J_{1}
\end{array}\right) .
\end{aligned}
$$

Since $J_{2} S_{1} J_{1}=J_{1} S_{1} J_{2}=J_{2} S_{i} J_{1}=J_{1} S_{i} J_{2}=0$, each of the matrices $S_{1}$ and $S_{i}$ is a direct sum of a matrix in $M_{c_{1}}$ and a matrix in $M_{c_{2}}$. Furthermore, we can conclude that $S_{1}=S_{i}=P_{1} \oplus P_{2}$, where $P_{1} \in M_{c_{1}}$ and $P_{2} \in M_{c_{2}}$, from $S_{1} J_{1}-J_{1} S_{i}=0=S_{1} J_{2}-J_{2} S_{i}$. Since $i$ is arbitrary, $S S^{*}=I_{m} \otimes\left(P_{1} \oplus P_{2}\right)$ where $P_{1}$ and $P_{2}$ are both positive definite. Thus there exist unitary $U_{1} \in M_{c_{1}}$ and $U_{2} \in M_{c_{2}}$ such that $U_{1} P_{1} U_{1}^{*}=D_{1}$ and $U_{2} P_{2} U_{2}^{*}=D_{2}$, where $D_{1}$ and $D_{2}$ are diagonal matrices with positive diagonal entries.

Let $U=I_{m} \otimes\left(U_{1} \oplus U_{2}\right)$ and $\tilde{S}=U S$. Then $\tilde{S} \tilde{S}^{*}=I_{m} \otimes\left(D_{1} \oplus D_{2}\right)$. Since the row vectors of $\tilde{S}$ form an orthogonal basis, we may write $\tilde{S}=D V$, where $D=I_{m} \otimes\left(D_{1} \oplus D_{2}\right)^{1 / 2}$ and $V$ is unitary.

On the other hand, we have $U^{*} B U=B$ for the block matrix $B$ in ([6), since

$$
a_{u v} I_{c_{1}} \oplus a_{v u} I_{c_{2}}=\left(U_{1} \oplus U_{2}\right)^{*}\left(a_{u v} I_{c_{1}} \oplus a_{v u} I_{c_{2}}\right)\left(U_{1} \oplus U_{2}\right) .
$$

Then $S^{*} B S=S^{*} U^{*} B U S=\tilde{S}^{*} B \tilde{S}=V^{*} D^{*} B D V$. In fact, the $(i, j)$-th block of $D^{*} B D$ is equal to

$$
\left(D_{1} \oplus D_{2}\right)^{1 / 2}\left(a_{u v} I_{c_{1}} \oplus a_{v u} I_{c_{2}}\right)\left(D_{1} \oplus D_{2}\right)^{1 / 2}=a_{u v} D_{1} \oplus a_{v u} D_{2} .
$$

Thus, $\phi$ has the asserted form. Since $\left\|I_{m} \otimes\left(D_{1} \oplus D_{2}\right)\right\|_{k^{\prime}}=\left\|\psi\left(I_{m}\right)\right\|_{r}=\left\|I_{m}\right\|_{k^{\prime}}=m$, it follows that $\operatorname{tr}\left(D_{1} \oplus D_{2}\right)=\operatorname{tr} D_{1}+\operatorname{tr} D_{2}=1$.

Finally, we consider case (III) $: m<n$. We prove the desired conclusion by induction on $n-m$ starting from $n-m=0$, which follows from cases (I) and (II). Suppose that $n-m=r>0$ and that the result holds for the cases when $n-m<r$. Applying the assumption on the restriction of $\phi$ on $M_{m, n}^{0}$, the subspace of $M_{m, n}$ that consists of matrices with zero $n$-th column, we conclude that for any $A \in M_{m, n}^{0}$,

$$
\phi(A)=U\left[\left(D_{1} \otimes \tilde{A}\right) \oplus\left(D_{2} \otimes \tilde{A}^{t}\right) \oplus 0_{p^{\prime}, q^{\prime}}\right] V
$$

where $\tilde{A}$ denotes the $m \times(n-1)$ matrix obtained from $A$ by deleting the $n$-th column, $\left(p^{\prime}, q^{\prime}\right)=\left(p-c_{1} m-c_{2}(n-1), q-c_{1}(n-1)-c_{2} m\right), U \in M_{p}$ and $V \in M_{q}$ are unitary, and the following holds:

(a) if $k^{\prime}<m$ and $c=c_{1}+c_{2}=k / k^{\prime}$, then $D_{1}=\frac{1}{c} I_{c_{1}}$ and $D_{2}=\frac{1}{c} I_{c_{2}}$;

(b) if $k^{\prime}=m$ and $c=c_{1}+c_{2} \leq k / k^{\prime}$, then $D_{1} \in M_{c_{1}}$ and $D_{2} \in M_{c_{2}}$ are diagonal matrices with positive diagonal entries such that $\operatorname{tr} D_{1}+\operatorname{tr} D_{2}=1$.

Now replacing $\phi$ by $X \mapsto U^{*} \phi(X) V^{*}$, we may assume that $U=I_{p}$ and $V=I_{q}$.

For any $\mathrm{x} \in M_{m, 1}$, let $A$ be the $m \times n$ matrix with $\mathbf{x}$ as the $n$-th column and zero in the other columns, and $X=\left(X_{u v}\right)_{1 \leq u, v \leq c+1}=\phi(A)$, where $X_{u u} \in M_{m, n-1}$ for $1 \leq u \leq c_{1}, X_{u u} \in M_{n-1, m}$ for $c_{1}<u \leq c$ and $X_{c+1, c+1} \in M_{p^{\prime}, q^{\prime}}$.

Take any nonzero $\mathbf{y} \in M_{m, 1}$ such that $\mathbf{x}^{*} \mathbf{y}=0$. (Note that $1<k \leq m$ and hence $\mathbf{y}$ exists.) For any $l<n$, let $B$ be the $m \times n$ matrix with $\mathbf{y}$ as the $l$-th column and zero in all other columns. Then $Y=\phi(B)=\left(D_{1} \otimes \tilde{B}\right) \oplus\left(D_{2} \otimes(\tilde{B})^{t}\right) \oplus 0_{p^{\prime}, q^{\prime}}$. 
Since $A$ and $B$ are orthogonal, $X^{*} Y=0_{q}$ and $X Y^{*}=0_{p}$. It follows from the structure of $Y$ that

$$
\begin{aligned}
X_{u v}^{*} \tilde{B} & =0 \text { when } 1 \leq u \leq c_{1} \text { and } 1 \leq v \leq c+1, \\
X_{u v}^{*} \tilde{B}^{t}=0 & \text { when } \quad c_{1}<u \leq c \text { and } 1 \leq v \leq c+1, \\
X_{u v} \tilde{B}^{*} & =0 \text { when } 1 \leq u \leq c+1 \text { and } 1 \leq v \leq c_{1}, \\
X_{u v}\left(\tilde{B}^{t}\right)^{*} & =0
\end{aligned}
$$

Since the $l$-th column of the $m \times(n-1)$ matrix $\tilde{B}$ is the nonzero vector $y$, if $X_{u v} \tilde{B}^{*}=0$, then the $l$-th row of $X_{u v}$ must be the zero vector. Furthermore, since $l$ can be any integer in $\{1, \ldots, n-1\}$, we conclude that $X_{u v}=0$. Similarly, $X_{u v}$ must be the zero matrix if $X_{u v}^{*} \tilde{B}^{t}=0$.

On the other hand, if $X_{u v}^{*} \tilde{B}=0$, then all the columns of $X_{u v}$ must be orthogonal to $\mathbf{y}$. Since $\mathbf{y}$ can be any vector orthogonal to $\mathbf{x}$, all columns of $X_{u v}$ must be multiples of $\mathbf{x}$. Hence, $X_{u v}=\mathbf{x w}^{t}$ for some vector $\mathbf{w}$ of suitable size. Similarly, since $X_{u v}\left(\tilde{B}^{t}\right)^{*}=0$, we have $X_{u v}=\mathbf{z x}^{t}$ for some $\mathbf{z}$.

By the arguments in the last two paragraphs, if $1 \leq u \leq c_{1}$ and $c_{1}<v \leq c$, then $\mathbf{x w}^{t}=X_{u v}=\mathbf{z} \mathbf{x}^{t}$ for some $\mathbf{w}$ and $\mathbf{z}$ of suitable sizes. Thus, $\mathbf{w}=\lambda \mathbf{x}$ for some constant $\lambda$ in $\mathbb{C}$, that is, $X_{u v}=\lambda \mathbf{x} \mathbf{x}^{t}$.

Combining the above analysis, we know that

$$
\phi\left[0_{m, n-1} \mid \mathbf{x}\right]=\left(\begin{array}{ccc}
0_{c_{1} m, c_{1} n} & E(\mathbf{x}) & F(\mathbf{x}) \\
0_{c_{2} n, c_{1} n} & 0_{c_{2} n, c_{2} m} & 0_{c_{2} n, q^{\prime}} \\
0_{p^{\prime}, c_{1} n} & G(\mathbf{x}) & H(\mathbf{x})
\end{array}\right)
$$

where

$$
\begin{aligned}
& E(\mathbf{x})=\left(\lambda_{u v} \mathbf{x x}^{t}\right)_{1 \leq u \leq c_{1}, 1 \leq v \leq c_{2},} \\
& F(\mathbf{x})=\left(\begin{array}{c}
\mathbf{x w}_{1}^{t} \\
\vdots \\
\mathbf{x w}_{c_{1}}^{t}
\end{array}\right), \\
& G(\mathbf{x})=\left(\begin{array}{lll}
\mathbf{z}_{1} \mathbf{w}^{t} & \cdots & \mathbf{z}_{c_{2}} \mathbf{x}^{t}
\end{array}\right),
\end{aligned}
$$

$H(\mathbf{x}), \lambda_{u v}, \mathbf{w}_{u}$ and $\mathbf{z}_{v}$ all depend on $\mathbf{x}$. By linearity of $\phi, \lambda_{u v}, \mathbf{w}_{u}$ and $\mathbf{z}_{v}$ must be the same for all $\mathbf{x}$, and $\lambda_{u v}$ must be zero. i.e., $E(\mathbf{x})=0_{c_{1} m, c_{2} m}$.

Now we consider the orthogonal pair $A=E_{11}+E_{1 n}$ and $B=-E_{21}+E_{2 n}$. Let $\mathbf{e}_{i}$ be the $i$-th column of $I_{m}$. Then

$$
\phi(A)=\left(\begin{array}{ccc}
D_{1} \otimes \tilde{E}_{11} & 0_{c_{1} m, c_{2} m} & F\left(\mathbf{e}_{1}\right) \\
0_{c_{2} n, c_{1} n} & D_{2} \otimes \tilde{E}_{11}^{t} & 0_{c_{2} n, q^{\prime}} \\
0_{p^{\prime}, c_{1} n} & G\left(\mathbf{e}_{1}\right) & H\left(\mathbf{e}_{1}\right)
\end{array}\right)
$$

and

$$
\phi(B)=\left(\begin{array}{ccc}
D_{1} \otimes-\tilde{E}_{21} & 0_{c_{1} m, c_{2} m} & F\left(\mathbf{e}_{2}\right) \\
0_{c_{2} n, c_{1} n} & D_{2} \otimes-\tilde{E}_{21}^{t} & 0_{c_{2} n, q^{\prime}} \\
0_{p^{\prime}, c_{1} n} & G\left(\mathbf{e}_{2}\right) & H\left(\mathbf{e}_{2}\right)
\end{array}\right) .
$$




$$
\begin{aligned}
& \text { Set } W=\left(\begin{array}{c}
\mathbf{w}_{1}^{t} \\
\vdots \\
\mathbf{w}_{c_{1}}^{t}
\end{array}\right) \text {. Since } \phi(A) \phi(B)^{*}=0 \text {, the }(1,1) \text {-th block equals } \\
& \qquad \begin{aligned}
0_{c_{1} m} & =\left(D_{1} \otimes \tilde{E}_{11}\right)\left(D_{1} \otimes-\tilde{E}_{21}\right)^{*}+F\left(\mathbf{e}_{1}\right) F\left(\mathbf{e}_{2}\right)^{*} \\
& =-\left(D_{1}^{2} \otimes E_{12}\right)+\left(W W^{*} \otimes E_{12}\right) \\
& =\left(W W^{*}-D_{1}^{2}\right) \otimes E_{12} .
\end{aligned}
\end{aligned}
$$

Thus, $W W^{*}=D_{1}^{2}$. Let $D_{1}=\operatorname{diag}\left(d_{1}, \ldots, d_{c_{1}}\right)$. Hence, $\left\{\mathbf{w}_{1} / d_{1}, \ldots, \mathbf{w}_{c_{1}} / d_{c_{1}}\right\}$ is a set of orthonormal vectors. Let $U \in M_{q^{\prime}}$ be a unitary matrix with $\mathbf{w}_{1}^{t} / d_{1}, \ldots$, $\mathbf{w}_{c_{1}}^{t} / d_{c_{1}}$ as the first $c_{1}$ rows. Then $F^{\prime}(\mathbf{x})=F(\mathbf{x}) U^{*}=\left[D_{1} \otimes \mathbf{x} \mid 0_{c_{1} m, q^{\prime}-c_{1}}\right]$.

Similarly, by considering $\phi(A)^{*} \phi(B)=0$, we write

$$
G^{\prime}(\mathbf{x})=V^{*} G(\mathbf{x})=\left(\begin{array}{c}
D_{2} \otimes \mathbf{x}^{t} \\
0_{p^{\prime}-c_{2}, c_{2} m}
\end{array}\right)
$$

for some unitary $V$. Now, we write

$$
\phi\left[0_{m, n-1} \mid \mathbf{x}\right]=\left(I_{c n} \oplus V\right)\left(\begin{array}{ccc}
0_{c_{1} m, c_{1} n} & 0_{c_{1} m, c_{2} m} & F^{\prime}(\mathbf{x}) \\
0_{c_{2} n, c_{1} n} & 0_{c_{2} n, c_{2} m} & 0_{c_{2} n, q^{\prime}} \\
0_{p^{\prime}, c_{1} n} & G^{\prime}(\mathbf{x}) & H^{\prime}(\mathbf{x})
\end{array}\right)\left(I_{c n} \oplus U\right) .
$$

On the other hand, by applying the assumption on the restriction of $\phi$ on the subspace of $M_{m, n}$ that consists of matrices with zero in the $(n-1)$-th column, we conclude that

$$
\operatorname{rank} \phi\left[0_{m, n-1} \mid \mathbf{x}\right]=\operatorname{rank} \phi\left[\mathbf{x}\left|0_{m, n-2}\right| \mathbf{x}\right]=\operatorname{rank} \phi\left[\mathbf{x} \mid 0_{m, n-1}\right]=c .
$$

(Note that here we used the fact that $n>m \geq 2$ to ensure nontrivial consideration.) Therefore, $H^{\prime}(\mathbf{x})=0$ for all $\mathbf{x}$. Finally, there exist permutation matrices $P$ and $Q$ such that for $A=\left[0_{m, n-1} \mid \mathbf{x}\right]$,

$$
\phi(A)=\left(I_{c n} \oplus V\right) P\left[\left(D_{1} \otimes A\right) \oplus\left(D_{2} \otimes A^{t}\right) \oplus 0_{p^{\prime}-c_{2}, q^{\prime}-c_{1}}\right] Q\left(I_{c n} \oplus U\right) .
$$

The result follows.

\section{REFERENCES}

[1] R. Bhatia, Matrix Analysis, Springer-Verlag, New York, 1997. MR 98i:15003

[2] W. S. Cheung and C. K. Li, Linear maps transforming the unitary group, Bulletin Canad. Math. Soc. 46 (2003), 54-58. MR 2003m:15007

[3] W. S. Cheung, C. K. Li and Y. T. Poon, Isometries between matrix algebras, to appear in J. Australian Math. Soc. pdf file available at http://www.resnet.wm.edu/ cklixx/poon-7.pdf.

[4] R. Grone and M. Marcus, Isometries of matrix algebras, J. Algebra 47 (1977), 180-189. MR 56:3039

[5] R. V. Kadison, Isometries of operator algebras, Ann. of Math. (2) 54 (1951), 325-338. MR $13: 256 \mathrm{a}$

[6] C. K. Li, Matrices with some extremal properties, Linear Algebra Appl. 101 (1988), 255-267. MR 89c:15025 
[7] C. K. Li, L. Rodman, and P. Šemrl, Linear transformations between matrix spaces that map one rank specific set into another, Linear Algebra Appl. 357 (2002), 197-208. MR 2003i:15002

[8] C. K. Li, P. Šemrl, and A. R. Sourour, Isometries for Ky-Fan norms on block triangular matrix algebras, Archiv Math. 81 (2003), 175-181.

Department of Mathematics, College of William and Mary, P.O. Box 8795, WilliamsBURG, VIRGINIA 23187-8795

E-mail address: ckli@math.wm.edu

Department of Mathematics, Iowa State University, Ames, Iowa 50011 - and - DepartMent of Mathematics, HKIEd, Hong Kong

E-mail address: ytpoon@ied.edu.hk

Department of Mathematics, The University of Hong Kong, Hong Kong

E-mail address: math2000@graduate.hku.hk 